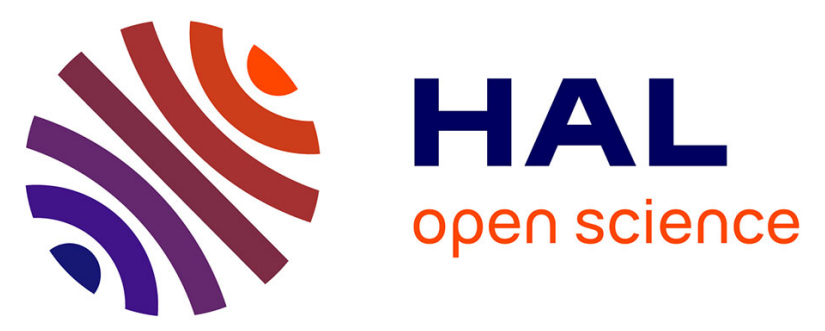

\title{
The Nationwide Austrian Registry: a prospective data collection on epidemiology; therapy and outcome of invasive mould infections in immunocompromised and/or immunosuppressed patients
}

S. Perkhofer, C. Lass-Flörl, M. Hell, G. Russ, R. Krause, M. Hönigl, C.

Geltner, J. Auberger, G. Gastl, M. Mitterbauer, et al.

\section{To cite this version:}

S. Perkhofer, C. Lass-Flörl, M. Hell, G. Russ, R. Krause, et al.. The Nationwide Austrian Registry: a prospective data collection on epidemiology; therapy and outcome of invasive mould infections in immunocompromised and/or immunosuppressed patients. International Journal of Antimicrobial Agents, 2010, 36 (6), pp.531. 10.1016/j.ijantimicag.2010.08.010 . hal-00640437

\section{HAL Id: hal-00640437 \\ https://hal.science/hal-00640437}

Submitted on 12 Nov 2011

HAL is a multi-disciplinary open access archive for the deposit and dissemination of scientific research documents, whether they are published or not. The documents may come from teaching and research institutions in France or abroad, or from public or private research centers.
L'archive ouverte pluridisciplinaire HAL, est destinée au dépôt et à la diffusion de documents scientifiques de niveau recherche, publiés ou non, émanant des établissements d'enseignement et de recherche français ou étrangers, des laboratoires publics ou privés. 


\section{Accepted Manuscript}

Title: The Nationwide Austrian Aspergillus Registry: a prospective data collection on epidemiology; therapy and outcome of invasive mould infections in immunocompromised and/or immunosuppressed patients

Authors: S. Perkhofer, C. Lass-Flörl, M. Hell, G. Russ, R.



Krause, M. Hönigl, C. Geltner, J. Auberger, G. Gastl, M.

Mitterbauer, B. Willinger, P. Knöbl, G. Resch, R. Waldner, A.

Makrai, G. Hartmann, M. Girschikofsky, R. Greil

PII:

S0924-8579(10)00369-9

DOI: doi:10.1016/j.ijantimicag.2010.08.010

Reference:

ANTAGE 3416

To appear in:

International

Journal

of

Antimicrobial

Agents

Received date: $\quad 14-7-2010$

Revised date: $\quad 3-8-2010$

Accepted date: $\quad 3-8-2010$

Please cite this article as: Perkhofer S, Lass-Flörl C, Hell M, Russ G, Krause R, Hönigl M, Geltner C, Auberger J, Gastl G, Mitterbauer M, Willinger B, Knöbl P, Resch G, Waldner R, Makrai A, Hartmann G, Girschikofsky M, Greil R, The Nationwide Austrian Aspergillus Registry: a prospective data collection on epidemiology; therapy and outcome of invasive mould infections in immunocompromised and/or immunosuppressed patients, International Journal of Antimicrobial Agents (2010), doi:10.1016/j.ijantimicag.2010.08.010

This is a PDF file of an unedited manuscript that has been accepted for publication. As a service to our customers we are providing this early version of the manuscript. The manuscript will undergo copyediting, typesetting, and review of the resulting proof before it is published in its final form. Please note that during the production process errors may be discovered which could affect the content, and all legal disclaimers that apply to the journal pertain. 
The Nationwide Austrian Aspergillus Registry: a prospective data collection on epidemiology, therapy and outcome of invasive mould infections in immunocompromised and/or immunosuppressed patients
S. Perkhofer ${ }^{a}$, C. Lass-Flörl ${ }^{a, *}$, M. Hell ${ }^{b}$, G. Russ ${ }^{c}$, R. Krause ${ }^{d}$, M. Hönigl ${ }^{d}$, C.

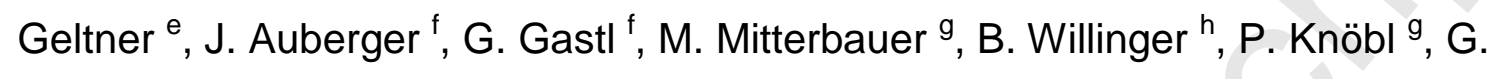 Resch $^{\text {', R. Waldner }}{ }^{\text {j, }}$ A. Makrai ${ }^{\text {j, G. Hartmann }}{ }^{\text {k, }}$ M. Girschikofsky ', R. Greil ${ }^{\text {c }}$

a Division of Hygiene and Medical Microbiology, Innsbruck Medical University, Fritz Pregl Str. 3/III, 6020 Innsbruck, Austria

${ }^{\mathrm{b}}$ Hospital Hygiene, Medical University of Salzburg, Salzburg, Austria

c IIIrd Medical Department with Hematology, Medical Oncology, Hemostaseology, Rheumatology and Infectious Diseases, Laboratory of Immunological and Molecular Cancer Research, Private Medical University Hospital Salzburg, Salzburg, Austria

' Internal Medicine, Graz Medical University, Graz, Austria

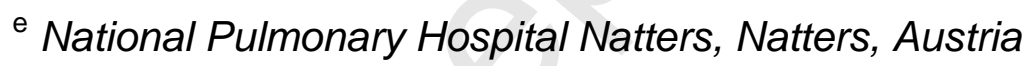

${ }^{f}$ Department of Haematology and Oncology, Innsbruck Medical University, Innsbruck, Austria

${ }^{9}$ Department of Haematology and Oncology, Vienna Medical University, Vienna, Austria

${ }^{\mathrm{h}}$ Department of Medical Microbiology, Vienna Medical University, Vienna, Austria

' Internal Medicine, Klinikum Wels-Grießkirchen, Wels, Austria

${ }^{j}$ Hanusch Hospital, Vienna, Austria 


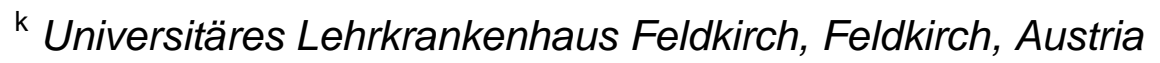

' Elisabethinen Hospital, Linz, Austria

ARTICLE INFO

Article history:

Received 14 July 2010

Accepted 3 August 2010

Keywords:

Austrian National Registry

Aspergillosis

Zygomycosis

Leukaemia

Transplantation

Antifungal treatment

* Corresponding author. Tel.: +43 5129003 70703; fax: +43 512900373700.

E-mail address: cornelia.lass-floerl@i-med.ac.at (C. Lass-Flörl). 


\section{ABSTRACT}

A prospective, observational, multicentre study was performed to assess the incidence, diagnosis, epidemiology and outcome of invasive mould infections (IMIs) reported to the Nationwide Austrian Aspergillus Registry. In total, 186 cases were recorded, corresponding to an annual incidence of 42 cases/1000 patients at risk or 2.36 cases/100 000 inhabitants. Patients with acute myelogenous leukaemia (34\%) and lung transplant recipients (17\%) are currently at highest risk for IMI, followed by a mixed population with impaired immunity (14\%). In total, $34 \%, 30 \%$ and $36 \%$ were proven, probable and possible cases of IMI. Predominant pathogens were Aspergillus spp. (67\%), followed by the zygomycetes (28\%). Voriconazole was the most frequently administered agent (38\%), followed by caspofungin $(20 \%)$ and posaconazole (19\%). Eighty patients $(43 \%)$ received antifungal prophylaxis for $\geq 7$ days, $30 \%$ of whom (24 patients) suffered from a breakthrough infection. The overall crude 12 -week mortality was $34 \%$. Multivariate analysis showed that outcome and survival did not correlate with the status of fungal disease, breakthrough infection, fungal species or age $(P>0.05)$. Aspergillosis remains the most commonly identified IMI among immunocompromised and/or immunosuppressed patients, but other moulds constitute a significant problem. Survival from IMls appears to have improved and the main challenge is to overcome breakthrough fungal infections. 


\section{Introduction}

Immunocompromised and immunosuppressed patients have a high risk of acquiring invasive fungal infections (IFIs) owing to cytopenia, B- and T-cell deficiencies specific to neoplastic diseases, as well as receipt of therapies to prevent and treat organ rejection in solid organ transplant recipients and for suppression of graft-versus-host disease in allogeneic stem cell or bone marrow recipients [1-5]. During the past two decades, the epidemiology of IFIs has changed: fluconazole prophylaxis has been successfully used to prevent Candida albicans infection [6], and mould infections have become more common [7]. This may be due to the fact that treatment of neoplastic diseases has changed substantially, including more aggressive therapies in patients with haematopoietic malignancies, the extension of the age range treated, e.g. with myelosuppressive or myeloablative therapies in acute leukaemia and myelodysplastic syndromes, and a higher number of subsequent lines of treatment for relapse, e.g. in many types of leukaemias and lymphomas [5,7-9]. These medical developments also apply to patients suffering from solid tumours. The trends and facts apparently lead to a prolongation of life in many of these neoplastic conditions, but simultaneously the time and severity of immunosuppression may also increase, thereby predisposing to the acquisition of IFI. In addition, changes in transplantation practices, including the sources used for stem cells, conditioning regimens and strategies to diagnose and treat IFI, have likely impacted the epidemiology and outcomes of infections $[8,9]$. Most recent data are limited to single-centre studies usually with a high patient volume and experience as well as a focus on allogeneic transplantation $[10,11]$. Few data are available on the incidence, prophylaxis and 
treatment of IFI in countries with a homogeneous health system and registries covering many centres with a different medical focus and specialisation.

The Nationwide Austrian Aspergillus Registry (NAAR) is such a prospective, multicentre, observational study group that collects data and monitors trends in the epidemiology, diagnosis, treatment and outcomes of invasive mould infections (IMIs) in Austria. This long-term initiative was undertaken with the overarching goal of shaping improvement of care for patients with IMIs and to assess the epidemiology and outcomes of IMls in Austria.

\section{Material and methods}

\subsection{Case identification and data collection}

The NAAR includes 12 major medical centres in Austria that have been chosen for participation based on the number of patients treated for IMI per year and the diversity of patients. Participating centres include care providers such as general oncology and/or haemato-oncology departments as well as stem cell and solid organ transplantation units. The registry collects data from routine clinical practice to address endpoints in four broad research areas of IMI, namely epidemiology, diagnosis, treatment and outcome. This study was performed by reviewing data from centres that reported at least four adults (age $\geq 19$ years) with an IMI during a 1-year period (2007-2008). The project is strictly observational, focusing on data collection. Patient data are kept anonymous and are entered prospectively into electronic case 
report forms. The registry has been approved by the Ethics Committee of Innsbruck Medical University, one of several lead ethics committees in Austria.

All patients with a diagnosis of proven, probable or possible IMI were eligible for enrolment according to the definitions of invasive fungal disease from the European Organization for Research and Treatment of Cancer/Invasive Fungal Infections Cooperative Group and the National Institute of Allergy and Infectious Diseases Mycoses Study Group (EORTC/MSG) Consensus Group [12]. Variables collected included demographic characteristics, underlying diseases, relevant and specific characteristics in case of transplantation, as well as type of IMI, treatment and outcome. Underlying diseases and characteristics included data on the type and state of haematological malignancy or other underlying disease as well as use of corticosteroids (within 30 days before the diagnosis) and other immunosuppressive agents. Furthermore, receipt of prior antifungal therapy (within 30 days before the diagnosis of infection), the fungal genus and species, diagnostic modalities, site of infection, history of antifungal therapy and therapeutic procedures were required to be reported. IMls were defined in accordance with published guidelines [12]. The therapeutic protocols used by the centres were similar and reflected current Infectious Diseases Society of America (IDSA) and/or European Conference on Infections in Leukemia (ECIL) guidelines $[13,14]$. The outcome of IMI therapy was recorded (i.e. 3-4 weeks after diagnosis) as complete or partial response, stable condition or worsening condition as assessed by investigators and as described elsewhere [11,15]. Breakthrough infection was defined as occurrence of proven fungal infection while on antifungal prophylaxis for at least 7 days. Survival was evaluated at 12 weeks. Each participating centre also contributed data on all newly 
diagnosed patients admitted during the survey period to gain an insight into the current incidence of IMI in the centres deemed representative of Austria.

After completion of an individual patient record, the record was locked and was available for inclusion in the clinical reports. For a complete patient record, the following requirements were defined in advance: (i) completion of follow-up for 12 weeks or until death or discharge; (ii) no outstanding data queries by the central coordinator(s) (C.L.-F. and J.A.); and (iii) a patient record sign-off by the site investigator. Participating sites also had the option to enter follow-up data at 12 months for patients whose records had been completed.

\subsection{Statistical analyses}

Comparison between categorical variables was performed by Fisher's exact test or $\chi^{2}$ test. Regression analysis with analysis of variance (ANOVA) was used to determine trends in incidence. A $P$-value of $<0.05$ was considered statistically significant.

\section{Results}

A total of 217 patients were prospectively enrolled and records for 186 patients were completed by January 2008. Baseline demographic characteristics and patient categories are described in Table 1 . The vast majority of infected patients suffered from haematological malignancies (68\%), with a smaller number of patients who had undergone allogeneic stem cell $(20 \%)$ or lung transplantation $(17 \%)$. The third most important group consisted of mixed individuals with and without known risk factors 
(14\%) (see Fig. 1). Underlying factors for this pooled group are prolonged Intensive Care Unit (ICU) stay associated with a high number of antibiotic drugs and/or need for ventilation, parenteral nutrition or central line access, neutrophil deficiencies or dysfunctions, and chronic granulomatous diseases. Overall, 89 patients (48\%) received treatment targeting T-cells and 56 patients (30\%) were treated with corticosteroids. Most of the culture-positive IMls (proven and probable) were caused by Aspergillus spp. (60 patients; $67 \%$ ), followed by zygomycetes (25 patients; $28 \%$ ). Infecting fungal pathogens are shown in Table 2, with Aspergillus fumigatus being the most frequently isolated species (59\%). Culture and microscopy was the most common diagnostic tool in identifying IMI in this series (64\%), followed by computed tomography (CT) (58\%). The diagnosis was proven in 63 cases (34\%) and was considered probable in 56 cases (30\%) (Table 3). The galactomannan assay [in serum and/or bronchoalveolar lavage fluid (BAL) specimens] was applied in $26 \%$ of cases, polymerase chain reaction (PCR) in $16 \%$ and the $\beta$-D-glucan assay in $3 \%$. Azoles, echinocandins and polyenes were used as single agents either sequentially or in combination. Voriconazole was the most commonly used antifungal agent (38\%), followed by caspofungin (20\%) and posaconazole (19\%). A minority of patients received itraconazole (1\%) or amphotericin B deoxycholate (4\%). Lipid formulations of amphotericin B plus posaconazole in combination were most frequently administered in the case of zygomycetes. Combination treatment, defined as more than one antifungal agent used sequentially or/and concomitantly, was administered in 67 patients (36\%). Treatment with voriconazole plus caspofungin was the most frequently used combination regimen (47 patients; 70\%). Eighty patients received antifungal prophylaxis for $\geq 7$ days (range 7-56 days, mean 21 days), of whom $30 \%$ (24 patients) had a breakthrough fungal infection. 
Posaconazole, voriconazole and itraconazole were the drugs (preferably orally) administered, and zygomycetes and $A$. fumigatus were the predominant pathogens. An adjunct procedure (e.g. surgical intervention) was performed in 6 patients (3\%). Overall, 93 patients (50\%) improved, 54 (29\%) were stable and 39 (21\%) deteriorated; the 12-week assessment estimated a mortality rate of $34 \%$ (see Table 3). In total, 186 cases of IMI were recorded, which corresponds to an annual incidence of 42 cases/1000 patients at risk or to 2.36 cases/100 000 inhabitants. Multivariate analysis showed that outcome and survival did not correlate with the status of fungal disease, breakthrough infection, fungal species or age $(P>0.05)$. Combination treatment, applied as first line in $19 \%$ of cases, was not superior compared with monotherapy. Survival and outcome were superior with voriconazole compared with the other drugs $(P<0.01)$ (see Fig. 2).

\section{Discussion}

This prospective, observational, multicentre study was performed to evaluate the contemporary incidences, epidemiological characteristics, clinical presentations and outcomes of IMls in 186 adults during the period 2007-2008. Restriction to proven and probable infections recorded an annual incidence of 19 cases/1000 patients at risk and 1.08 cases/100 000 inhabitants for Austria. Patients with haematological malignancies and organ transplant recipients were at highest risk. The third most important group consisted of a mixed population with various underlying risk factors, including mainly ICU patients. Important findings also included an improvement in IMI-associated mortality, although there was a considerable rate of breakthrough fungal infections. 
The NAAR is a network of medical institutions gathering and sharing information to increase understanding of the incidence, epidemiology, therapy and outcomes of IMls in Austria.

The ability to capture and display information on demographics, epidemiology, treatment and outcomes of patients from multiple clinical centres greatly enhances the timely dissemination of accurate and detailed clinical information and current approaches to therapy.

In this analysis, invasive aspergillosis was the most commonly encountered IMI, presenting predominantly as pneumonia in patients with haematological malignancies and as tracheobronchitis in organ transplant recipients. The second most important fungi were the zygomycetes, followed by a small number of uncommon moulds. Occurrence of infections due to zygomycetes was not focused on a single centre, but rather was reported from several institutions. This trend, first described in patients who received transplants after 1995, probably reflects the inclusion of higher risk patients in modern tumour therapies, the increasing number of subsequent lines of antineoplastic treatment applied, and the better tolerability of treatment by young olds and older olds with an increasing number of patients at risk, as well as by the increased use of higher rates of chemoimmunotherapies. In addition, higher risk patients are undergoing stem cell transplantation with incompletely matches donors leading to a more immunosuppressed patient population in this group. Moreover, extensive use of prophylactic treatment with agents that have activity against Aspergillus spp. $[8,14,16,17]$ may have favoured a 
shift towards zygomycoses. So far, zygomycoses were observed mainly in tertiary care centres, showing obvious differences in patients at risk or in clinical approaches. Within the setting of organ transplantation recipients, this epidemiological trend was strongest, displaying the highest incidence (8\%) with rare mould infections. Overall, incidences of IMI are comparable with other studies, showing patients with acute myeloid leukaemia being at highest risk for IMI $[18,19]$. The third most important group at risk consists of mixed individuals with various types of underlying immunodeficiency. Patients with prolonged ICU stay and use of steroids, neutrophil deficiencies or dysfunctions, and chronic granulomatous diseases are an emerging group for IMI and are definitively on the rise. In this patient population, most of the group had no underlying haematological malignancies and suffered from a primary mould infection.

Culture and microscopy was the most common diagnostic tool in identifying IMIs in this series (64\%). In several Austrian centres, we attempt to perform CT-guided percutaneous lung biopsies to confirm the diagnosis [20]. This intervention appears to be mandatory in our patient population, as we record a high rate of infections due to Aspergillus terreus and zygomycetes [21]. Aspergillus terreus is amphotericin Bresistant yet this drug is mandatory for zygomycoses. A similar diagnostic approach is widely used in other Austrian institutions. The diagnosis was proven in 63 cases $(34 \%)$ and probable in 56 cases (30\%). However, there is a need to improve diagnostic approaches, as only a limited number of other diagnostic tests were used to establish early diagnosis. The galactomannan assay (in serum and/or BAL specimens) was applied in $26 \%$ of cases, PCR in $16 \%$ and the $\beta$-D-glucan assay in 
$3 \%$. Based on the relatively high rate of breakthrough infections in patients receiving prophylaxis, this approach might be helpful for timely and sensitive diagnosis.

The outcome of current treatment regimens (3-4 weeks after diagnosis) was satisfactory compared with other studies [22-24]. Overall, 50\% of patients improved, $29 \%$ were stable and $21 \%$ deteriorated. Of note, conventional amphotericin B was minimally used in this study. As this reflects practice observed in 12 different medical centres, it appears that conventional amphotericin B has largely been replaced by lipid formulations of amphotericin B and the new third-generation azoles.

Combination therapy, either sequential or concomitant, was frequently observed in $36 \%$ of patients. This reflects the real-life situation and possibly reflects the high rate of breakthrough infections observed. However, combination treatment was not superior for outcome and/or survival compared with single drug use. Voriconazole appears to be associated with improved outcome and survival $(P<0.01)$; a similar trend for outcome was for posaconazole.

Here we report a 12 -week mortality rate of $34 \%$ among patients with IMI, which is markedly improved compared with rates from prior reports of outcomes during the 1990s [25-27]. These data on aspergillosis are consistent with recently reported data $[1,28,29]$. Improved outcomes likely reflect improved diagnostic and therapeutic modalities compared with older patient series. However, in these studies mortality was highest in patients with zygomycoses [1]. This is in contrast to the present report where IMI-related deaths occurred to the same extent for infections due to Aspergillus and zygomycetes. 
Outcome and survival did not correlate with the underlying fungal disease status, yet it was significantly better in patients receiving voriconazole. The reason might lay in the fact that most of the infections were due to $A$. fumigatus and that this azole has excellent anti-Aspergillus activity [24]. Moreover, voriconazole was primarily used as first-line treatment and other drugs as rescue.

However, in this registry, with all the limitations of its nature, it has been shown that breakthrough IMIs occurred frequently in patients receiving antifungal prophylaxis. Aspergillus and zygomycetes were the main organisms, however the reason for this high rate $(30 \%)$ of breakthrough infection is not clear yet and several causes might be responsible. First, the distinction between baseline infections (those present on or immediately before starting treatment) and breakthrough infections (those with an onset while on treatment) may be difficult to ascertain [30]. Second, serum drug concentrations may be insufficient to prevent infections [31] as patients do not follow the strict guidelines for oral drug uptake. Third, diagnosis has improved to such an extent and allows for more sensitive and timely diagnosis and more tailored therapy [32]. In an earlier study, it was shown that $>50 \%$ of infections were identified at autopsy [33]. So far, breakthrough aspergillosis in patients receiving empirical or prophylactic antifungal treatment using an agent with anti-Aspergillus activity is well known. For example, breakthrough aspergillosis was observed in 1.0-18\% of patients receiving conventional amphotericin $B$, lipid-based amphotericin $B$, voriconazole or itraconazole and caspofungin [30,34-39]. Recently, a rate of breakthrough invasive aspergillosis among patients receiving caspofungin for persistent fever and neutropenia was recorded as 13\% [35]. However, statements are somewhat limited on this topic as we have no access to the overall data on how 
many patients received successful prophylaxis. None the less, our Austrian-wide rate of breakthrough infection needs to be investigated in more detail. So far, breakthrough infections while on an azole (e.g. posaconazole) did not exclude beneficial treatment with another azole (e.g. voriconazole).

Other registries or studies have also gathered information on IMIs but have been hindered by a scope of observations limited in terms of patient type, therapies used or geographic areas $[7,10,11]$. In contrast, the NAAR is a registry that allows the collection and analysis of high-quality data obtained from targeted centres with substantial clinical experience and is facilitated by the use of electronic data capture and reporting software. The wide distribution of investigating centres across Austria, the variety of patient categories and the enrolment of more than 150 patients with IMI per year make this registry a significant tool for the study of common, rare and emerging IMIs.

This registry has some limitations. Because this is a strictly observational project, the generated results might have limited clinical significance. It is likely that patients with possible infections are underrepresented in the database because of the challenges in ascertainment. Despite its limitations, the NAAR is a network of medical institutions collecting significant information regarding IMIs in Austria. As enrolment continues, additional data will be analysed and published, which will provide valuable information regarding the epidemiology, therapy and outcomes of IMIs.

In conclusion, this study shows that aspergillosis remains the most commonly identified IMI among immunocompromised and/or immunosuppressed patients, but 
other moulds constitute a significant problem. Survival from IMIs appears to have improved compared with previous reported data. Overall, the main challenge is to overcome breakthrough fungal infections. A mixed patient population with various underlying host defects comprise an emerging and important group for IMls.

\section{Funding}

C-LF has received research funding from Gilead, AESCA Pharma and Pfizer. SP has received research funding from Pfizer.

\section{Competing interests}

C-LF has served as a consultant or advisor to Gilead and Pfizer and has been on the speakers' bureau for Pfizer, Gilead and Merck Sharp \& Dohme. All other authors declare no competing interests.

\section{Ethical approval}

Research Ethics Committee of Innsbruck Medical University, Innsbruck, Austria (Judgement's reference no. UN2891). 


\section{References}

[1] Neofytos D, Horn D, Anaissie E, Steinbach W, Olyaei A, Fishman J, et al. Epidemiology and outcome of invasive fungal infection in adult hematopoietic stem cell transplant recipients: analysis of Multicenter Prospective Antifungal Therapy (PATH) Alliance registry. Clin Infect Dis 2009;48:265-73.

[2] Singh N, Paterson D. Aspergillus infections in transplant recipients. Clin Microbiol Rev 2005;18:44-69.

[3] Singh N. Trends in the epidemiology of opportunistic fungal infections: predisposing factors and the impact of antimicrobial use practices. Clin Infect Dis $2001 ; 33: 1692-6$.

[4] Segal B. Aspergillosis. N Engl J Med 2009;360:1870-84.

[5] Malani A, Kauffman C. Changing epidemiology of rare mould infections: implications for therapy. Drugs 2007;67:1803-12.

[6] Marr KA, Crippa F, Leisenring W, Hoyle M, Boeckh M, Balajee SA, et al. Itraconazole versus fluconazole for prevention of fungal infections in patients receiving allogeneic stem cell transplants. Blood 2004;103:1527-33.

[7] Marr AK, Carter R, Crippa F, Wald A, Corey L. Epidemiology and outcome of mould infections in hematopoietic stem cell transplant recipients. Clin Infect Dis 2002;34:909-17.

[8] Upton A, Marr AK. Emergence of opportunistic mould infections in the hematopoietic stem cell transplant patient. Curr Infect Dis Rep 2006;8:434-41.

[9] Patterson T. Advances and challenges in management of invasive mycoses. Lancet 2005;17:1013-25. 
[10] Lortholary O, Charlemagne A, Bastides F, Chevalier P, Datry A, Gonzalves MF, et al. A multicentre pharmacoepidemiological study of therapeutic practices in invasive fungal infections in France during 1998-1999. J Antimicrob Chemother 2004;54:456-64.

[11] Pappas P. Amphotericin B lipid complex in the treatment of invasive fungal infections: results of the Collaborative Exchange of Antifungal Research (CLEAR), an industry supported patient registry: introduction. Clin Infect Dis 2005;40(Suppl 6):S379-83.

[12] De Pauw B, Walsh TJ, Donnelly JP, Stevens DA, Edwards JE, Calandra T, et al. Revised definitions of invasive fungal disease from the European Organization for Research and Treatment of Cancer/Invasive Fungal Infections Cooperative Group and the National Institute of Allergy and Infectious Diseases Mycoses Study Group (EORTC/MSG) Consensus Group. Clin Infect Dis 2008;46:1813-21.

[13] Walsh TJ, Anaissie EJ, Denning DW, Herbrecht R, Kontoyiannis DP, Marr KA, et al. Treatment of aspergillosis: clinical practice guidelines of the Infectious Diseases Society of America. Clin Infect Dis 2009;46:327-60.

[14] Maertens J, Frere P, Lass-Flörl C, Heinz W, Cornely OA. Primary antifungal prophylaxis in leukaemia patients. Eur J Cancer 2007;Suppl 5:43-8.

[15] Post M, Lass-Flörl C, Gastl G, Nachbaur D. Invasive fungal infections in allogeneic and autologous stem cell transplant recipients: a single-center study of 166 transplanted patients. Transpl Infect Dis 2007;9:189-95.

[16] Almyroudis NG, Sutton DA, Linden P, Rinaldi MG, Fung J, Kusne S. Zygomycosis in solid organ transplant recipients in a tertiary transplant center and review of the literature. Am J Transplant 2006;6:2365-75. 
[17] Kontoyiannis DP, Lionakis MS, Lewis RE, Chamilos G, Healy M, Perego C, et al. Zygomycosis in a tertiary-care cancer center in the era of Aspergillus-active antifungal therapy: a case-control observational study of 27 recent cases. J Infect Dis 2005;191:1350-60.

[18] Chamilos G, Luna M, Lewis RE, Bodey GP, Chemaly R, Tarrand JJ, et al. Invasive fungal infections in patients with hematologic malignancies in a tertiary care cancer center: an autopsy study over a 15-year period (1989-2003). Haematologica 2006;91:986-9.

[19] Pagano L, Caira M, Candoni A, Offidani M, Fianchi L, Martino B, et al. The epidemiology of fungal infections in patients with hematologic malignancies: the SEIFEM-2004 study. Haematologica 2006;91:1068-75.

[20] Lass-Flörl C, Resch G, Nachbaur D, Mayr A, Gastl G, Auberger J, et al. The value of computed tomography-guided percutaneous lung biopsy for diagnosis of invasive fungal infection in immunocompromised patients. Clin Infect Dis 2007;45:e101-4.

[21] Lass-Flörl C, Griff K, Mayr A, Petzer A, Gastl G, Bonatti H, et al. Epidemiology and outcome of infections due to Aspergillus terreus: 10-year single centre experience. Br J Haematol 2005;131:201-7.

[22] Aisner J, Wiernik PH, Schimpff SC. Treatment of invasive aspergillosis: relation of early diagnosis and treatment to response. Ann Intern Med $1977 ; 86: 539-43$.

[23] Bodey G, Bueltmann B, Duguid W, Gibbs D, Hanak H, Hotchi M, et al. Fungal infections in cancer patients: an international autopsy study. Eur J Clin Microbiol Infect Dis 1992;11:99-109. 
[24] Herbrecht R, Denning DW, Patterson TF, Bennett JE, Greene RE, Oestmann JW, et al. Voriconazole versus amphotericin B for primary therapy of invasive aspergillosis. N Engl J Med 2002;347:408-15.

[25] Anaissie E. Opportunistic mycoses in the immunocompromised host: experience at a cancer center and review. Clin Infect Dis 1992;14(Suppl 1):S4353.

[26] Denning DW. Epidemiology and pathogenesis of systemic fungal infections in the immunocompromised host. J Antimicrob Chemother 1991;28:1-16.

[27] Pfaller MA. Epidemiological typing methods for mycosis. Clin Infect Dis 1992;14:4-10.

[28] Horn DL, Fishman JA, Steinbach WJ, Anaissie EJ, Marr KA, Olyaei AJ, et al. Presentation of the PATH Alliance registry for prospective data collection and analysis of the epidemiology, therapy, and outcomes of invasive fungal infections. Diagn Microbiol Infect Dis 2007;59:407-14.

[29] Auberger J, Lass-Flörl C, Ulmer H, Nogler-Semenitz E, Clausen J, Gunsilius $\mathrm{E}$, et al. Significant alterations in the epidemiology and treatment outcome of invasive fungal infections in patients with hematological malignancies. Int $\mathrm{J}$ Hematol 2008;88:508-15.

[30] Martino R, Viscoli C. Empirical antifungal therapy in patients with neutropenia and persistent or recurrent fever of unknown origin. Br J Haematol 2006;132:13854.

[31] Müller C, Arndt M, Queckenberg C, Cornely O, Theisohn M. HPLC analysis of the antifungal agent posaconazole in patients with haematological diseases. Mycoses 2006;49:17-22. 
[32] Lass-Flörl C. The changing face of epidemiology of invasive fungal diseases in Europe. Mycoses 2009;52:197-205.

[33] Groll AH, Shah PM, Menzel C, Schneider M, Just-Nuebling G, Huebner K. Trends in the postmortem epidemiology of invasive fungal infections at a university hospital. J Infect 1996;33:23-32.

[34] Trifilio SM, Bennett CL, Yarnold PR, McKoy JM, Parada J, Mehta J, et al. Breakthrough zygomycosis after voriconazole administration among patients with hematologic malignancies who receive hematopoietic stem-cell transplants or intensive chemotherapy. Bone Marrow Transplant 2007;39:425-9.

[35] Lafaurie M, Lapalu J, Raffoux E, Breton B, Lacroix C, Socié G, et al. High rate of breakthrough invasive aspergillosis among patients receiving caspofungin for persistent fever and neutropenia. Clin Microbiol Infect 2010;16:1191-6.

[36] Walsh TJ, Pappas P, Winston DJ, Lazarus HM, Petersen F, Raffalli J, et al. Voriconazole compared with liposomal amphotericin B for empirical antifungal therapy in patients with neutropenia and persistent fever. N Engl J Med 2002;346:225-34.

[37] Vehreschild JJ, Böhme A, Buchheidt D, Arenz D, Harnischmacher U, Heussel $\mathrm{CP}$, et al. A double-blind trial on prophylactic voriconazole (VRC) or placebo during induction chemotherapy for acute myelogenous leukaemia (AML). J Infect $2007 ; 55: 445-9$.

[38] Cornely OA, Maertens J, Winston DJ, Perfect J, Ullmann AJ, Walsh TJ, et al. Posaconazole vs. fluconazole or itraconazole prophylaxis in patients with neutropenia. N Engl J Med 2007;356:348-59. 
[39] Ullmann AJ, Lipton JH, Vesole DH, Chandrasekar P, Langston A, Tarantolo $\mathrm{SR}$, et al. Posaconazole or fluconazole for prophylaxis in severe graft-versus-host disease. N Engl J Med 2007;25:335-47. 
Fig. 1. Overview of underlying diseases in 186 patients with invasive mould infections. AML, acute myelogenous leukaemia; SOT, single-organ transplantation; VRF, various risk factors (mainly Intensive Care Unit patients without underlying haematological malignancies); NHL, non-Hodgkin's lymphoma; ALL, acute lymphatic leukaemia; MDS, myelodysplastic syndrome; CLL, chronic lymphatic leukaemia; ST, solid tumour.

Fig. 2. Distribution of antifungal agents used in 186 patients with invasive fungal infections. More than one antifungal agent may have been administered to one patient. * Outcome was superior with voriconazole compared with the other drugs applied $(P<0.01) .{ }^{*}$ Only four patients were investigated. L-Ampho B, lipid-based amphotericin B. 


\section{Table 1}

Baseline characteristics of 186 immunosuppressed patients with invasive mould infections $^{a}$

\begin{tabular}{|c|c|}
\hline Patient characteristic & $n(\%)$ \\
\hline Age (years) (mean \pm S.E.) & $52.9 \pm 0.6$ \\
\hline \multicolumn{2}{|l|}{ Age group (years) } \\
\hline$<18$ & $7(4)$ \\
\hline 19-39 & $26(14)$ \\
\hline $40-60$ & $82(44)$ \\
\hline$>60$ & $71(38)$ \\
\hline Male gender & $108(58)$ \\
\hline \multicolumn{2}{|l|}{ Underlying disease ${ }^{\mathrm{b}}$} \\
\hline Acute myelogenous leukaemia & $63(34)$ \\
\hline Acute lymphatic leukaemia & $17(9)$ \\
\hline Chronic lymphatic leukaemia & $10(5)$ \\
\hline Non-Hodgkin's lymphoma & $19(10)$ \\
\hline Hodgkin's lymphoma & $2(1)$ \\
\hline Multiple myeloma & $1(0.5)$ \\
\hline Myelodysplastic syndrome & $15(8)$ \\
\hline Solid tumour & $10(5)$ \\
\hline Lung transplant recipients & $31(17)$ \\
\hline Other & $18(10)$ \\
\hline Allogeneic stem cell transplantation & $37(20)$ \\
\hline \multicolumn{2}{|l|}{ Immunological risk factors ${ }^{b}$} \\
\hline Neutrophil count $<500$ cells $/ \mathrm{mm}^{3}$ & $80(43)$ \\
\hline Receipt of corticosteroid therapy & $56(30)$ \\
\hline Receipt of immunosuppressive therapy & $82(44)$ \\
\hline Inherited severe immunodeficiency & $6(3)$ \\
\hline \multicolumn{2}{|l|}{ Environmental conditions } \\
\hline HEPA rooms & $56(30)$ \\
\hline Exposure to construction (builder's dust) & $22(12)$ \\
\hline
\end{tabular}




\section{Standard rooms}

$108(58)$

Antifungal prophylaxis

$80(43)$

S.E., standard error; HEPA, high-efficiency particulate air.

${ }^{a}$ Data are number (\%) of patients, unless otherwise indicated.

${ }^{\mathrm{b}}$ Underlying diseases and immunological risk factors were not mutually exclusively (i.e. patients could have more than one factor). 


\section{Table 2}

Distribution of infecting fungal pathogens and species observed in patients with culture-positive invasive mould infections in Austria $(n=89)$

\begin{tabular}{ll}
\hline Pathogen & $\%$ \\
\hline Aspergillus spp. & 67 \\
Aspergillus fumigatus & 59 \\
Aspergillus flavus & 4 \\
Aspergillus terreus & 3 \\
Aspergillus niger & 1 \\
Zygomycetes & 28 \\
Absidia spp. & 10 \\
Mucor spp. & 9 \\
Rhizopus spp. & 9 \\
Other moulds & 4 \\
Penicillium spp. & 2 \\
\hline
\end{tabular}




\section{Table 3}

Clinical characteristics of 186 immunosuppressed patients with invasive mould infections

\begin{tabular}{ll}
\hline Characteristic & $n(\%)$ \\
\hline Diagnosis & $63(34)$ \\
Proven & $56(30)$ \\
Probable & $67(36)$ \\
Possible & $186(100)$ \\
Lung involvement & \\
Treatment outcome & $93(50)$ \\
Improvement & $54(29)$ \\
Stable & $39(21)$ \\
Deterioration & $80(43)$ \\
Prophylactic antifungal use ${ }^{\text {a }}$ & $24(30)$ \\
Breakthrough infection during prophylaxis & $123(66)$ \\
Survival at 12 weeks & $30(16)$ \\
Diagnostic screening assays & $48(26)$ \\
Any PCR & $6(3)$ \\
Serum/BAL galactomannan assay & \\
B-D-glucan assay & \\
\hline PCR, polymerase chain reaction; BAL, bronchoalveolar lavage fluid. \\
a Administration of an antifungal drug for at least 1 week before clinical signs and \\
symptoms of fungal infection. & \\
\hline
\end{tabular}




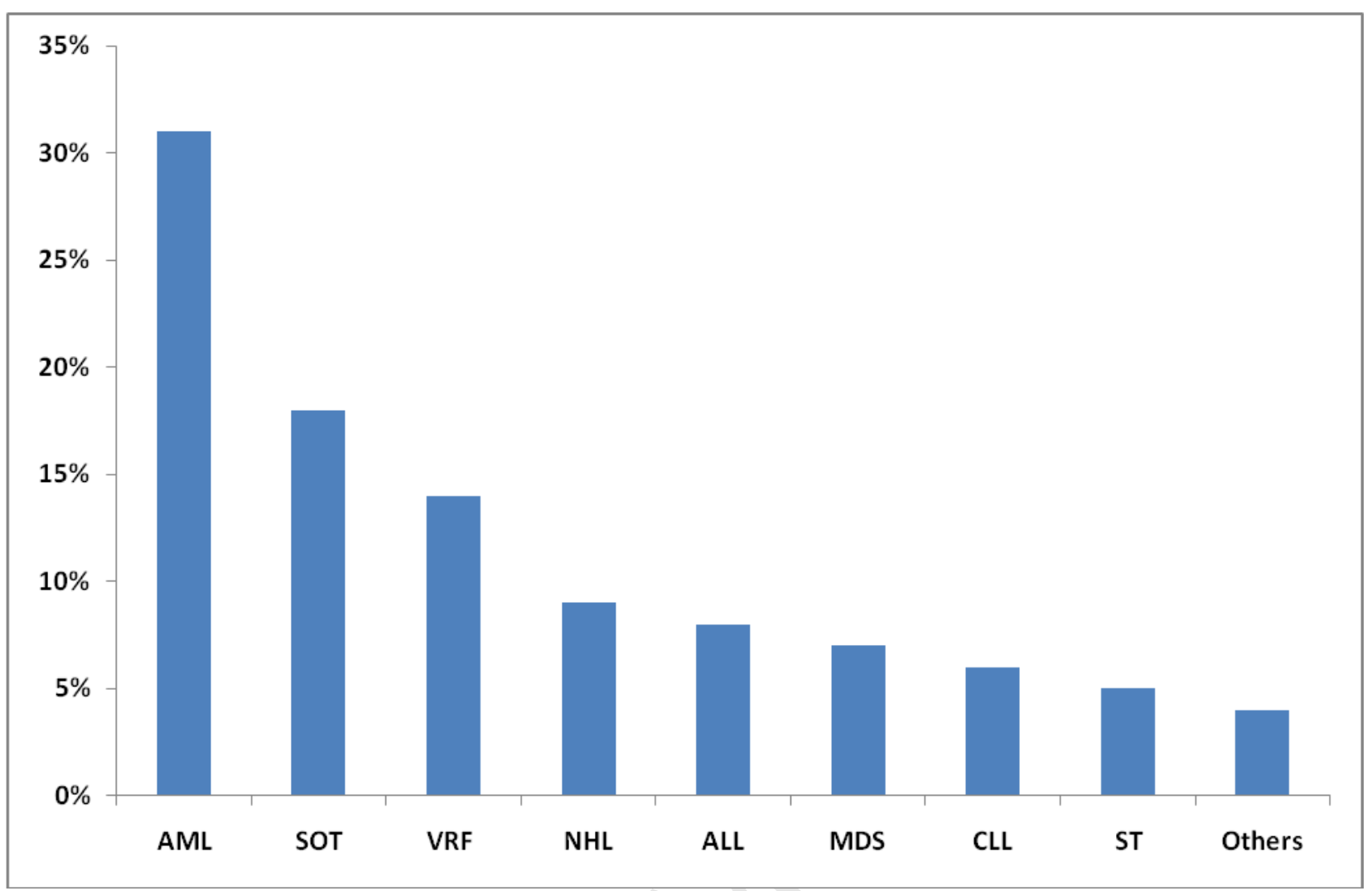

Page 27 of 28

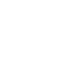


Edited Figure 2

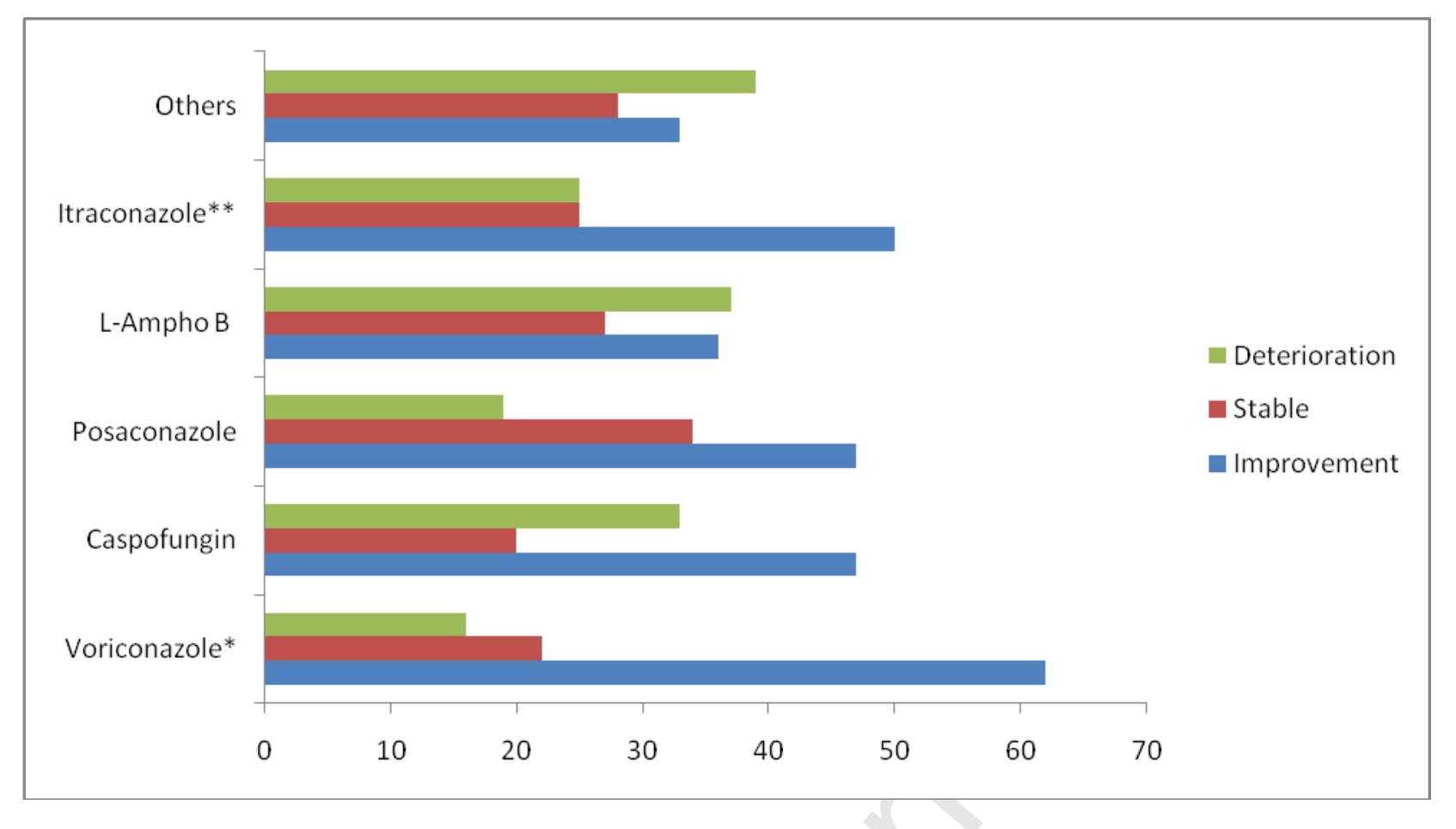

- Stable

- Improvement

Edited Figure 2 